\title{
Elbow medial collateral ligament injuries
}

\author{
Ra'Kerry K. Rahman · William N. Levine • \\ Christopher S. Ahmad
}

Published online: 6 June 2008

(C) The Author(s) 2008

\begin{abstract}
Elbow medial collateral ligament sprain occurs when the elbow is subjected to a valgus force exceeding the tensile properties of the medial collateral ligament (MCL). This is an injury seen more often in throwing athletes. Understanding the differential diagnosis of medial elbow pain is paramount to diagnose MCL injury as well as addressing other medial elbow pathology. A natural evolution regarding MCL injury has occurred over the past 20 years, with modifications of the original surgical procedure, specificity and sensitivity analysis of imaging modalities, and physical exam maneuvers to diagnose MCL pathology. In order for the MCL literature to advance further, more biomechanical and long-term clinical outcome data for the respective surgical modifications are needed. This review describes MCL injury pathophysiology, patient evaluation, reconstruction indications/contraindications, and current and evolving surgical techniques.
\end{abstract}

Keywords Elbow $\cdot$ Medial collateral ligament injury

\section{Introduction}

Elbow medial collateral ligament (MCL) sprain occurs when the elbow is subjected to a valgus, or laterally directed force, which distracts the medial side of the elbow, exceeding the tensile properties of the MCL. This is an injury seen more often in athletes, in particular overhead athletes, such as pitchers, javelin throwers, quarterbacks, tennis, volleyball, and water polo players. However, any elbow that

Ra'KerryK. Rahman · W. N. Levine · C. S. Ahmad ( $₫)$ Department of Orthopaedic Surgery, Center for Shoulder, Elbow and Sports Medicine, Columbia University Medical Center, 622 West 168th Street, PH 11th Floor, New York, NY 10032, USA

e-mail: csa4@columbia.edu experiences a sudden severe, valgus force can sustain an MCL injury-for example, this injury occurs frequently in wrestlers. MCL injuries can be acute or insidious. Regardless of the chronicity, these patients present with medial elbow pain. Understanding the differential diagnosis of medial elbow pain is paramount to diagnose MCL injury as well as addressing other medial elbow pathology.

MCL injuries were once difficult to diagnose, and surgical treatment was not available forcing athletes with these injuries to end their careers. The outlook for athletes with this injury changed after Jobe [1] developed the original MCL reconstruction, dubbed the "Tommy John procedure," in 1974. The success of this procedure which was published in 1986 encouraged the orthopedic and sports medicine community to refine the diagnostic approach as well as surgical technique involving MCL injury. As a result, an evolution regarding MCL injury has occurred over the past 20 years, with modifications of the original surgical procedure, improved imaging modalities, and new physical exam maneuvers to diagnose MCL pathology. There are many excellent reviews written in the recent past [2-7]. While new techniques have been developed and early results are encouraging, longer-term clinical outcome studies are needed. Furthermore, advances in MCL prevention strategies, particularly in the young athlete, are needed as this injury is being observed in the young athlete at an alarming rate [8]. This review describes MCL injury pathophysiology, patient evaluation, reconstruction indications/contraindications, and current and evolving surgical techniques.

\section{MCL injury pathophysiology}

The MCL complex consists of three ligaments: the anterior oblique (AOL), posterior oblique (POL), and transverse 
ligaments [9]. The AOL is the strongest component of the MCL and the primary restraint and stabilizer to stress of the medial elbow [10-12]. The AOL is functionally composed of anterior and posterior bands that provide a reciprocal function in resisting valgus stress through the range of flexion-extension motion [12,13]. Valgus stress is generated at the elbow during common throwing maneuvers in baseball, softball, football, tennis serving, and volleyball spiking. There are six phases of throwing: (i) wind up; (ii) early cocking; (iii) late cocking; (iv) acceleration; (v) deceleration; and (vi) follow through. (Fig. 1) The valgus stress is generated due to a combination of factors. The elbow can only flex and extend, no significant rotation occurs at the elbow. Therefore, as the shoulder internally rotates during acceleration, the flexed forearm is pulled forward. In this position, the lateral side of the elbow experiences compressive forces and the medial side experiences distraction forces. The calculated valgus torque created during the acceleration phase of throwing is very high. These estimated forces exceed the known ultimate tensile strength of cadaveric MCL specimens $(33 \mathrm{~N} \mathrm{~m})$ [14]. Thus the MCL is at obvious risk for injury from these repetitive forces. The flexor carpi ulnaris and flexor digitorum superficialis are the muscles which counteract the valgus stress during throwing. Thus, the muscular dynamic stability to the medial elbow is essential and must be included in injury prevention programs, postoperative rehabilitation programs, and morbidity from surgical techniques must be minimized.

Knowledge of the relationship between the posterior humerus and the anterior olecranon known as the posterior compartment of the elbow and the MCL is important in understanding the pathophysiology of MCL injury. Medial elbow pain is often observed in patients with posterior compartment pathology. The throwing motion may also result in posterior elbow pain as the olecranon is repeatedly and forcefully driven into the olecranon fossa. This shear force between the olecranon and the olecranon fossa can lead to the formation of posteromedial osteophytes and loose bodies and are common diagnoses leading to surgery in baseball players (Fig. 2) [2, 15, 16]. Andrews and Timmerman [16] reported that in a series of professional baseball players who underwent removal of loose bodies or osteophyte resection known as olecranon debridement, $25 \%$ developed valgus instability and eventually required MCL reconstruction. This observation suggests that both the olecranon and the MCL contribute to valgus stability. Additionally, there are studies which show increases in valgus angulation with stepwise resection of the olecranon.

\section{History and physical}

When evaluating a patient with medial elbow pain, the following etiologies should be considered: flexor-pronator tendon injury, MCL instability, valgus extension overload, ulnar neuropathy, ulnar nerve subluxation, medial triceps subluxation, medial epicondyle avulsion (skeletally immature), loose bodies, olecranon osteophytes, and medial antebrachial cutaneous nerve injury [2]. Patients with MCL injuries complain of medial elbow pain during the acceleration phase of throwing. Chronic injuries present gradually and often with pain occurring only when throwing $50-75 \%$ of maximal effort. Acute injuries may present suddenly with a pop, sharp pain, and inability to continue throwing. For patients with a component of valgus extension overload, elbow pain is also located on the posteromedial aspect of the olecranon and is present in both the acceleration and deceleration phases of throwing. Patients may report limited extension which results form impinging osteophytes. Athletes with associated ulnar neuritis report numbness and tingling of the little and ring fingers with repetitive throwing that is relieved with rest. Athletes with symptomatic loose bodies may complain of catching or locking of the elbow that at times can be released with elbow manipulation.

Physical examination features suggesting MCL injury include point tenderness directly over the MCL or toward its insertion sites. Valgus instability is tested with the patients' elbow flexed between 20 and $30^{\circ}$ to unlock the
Fig. 1 The throwing phases during a baseball pitch

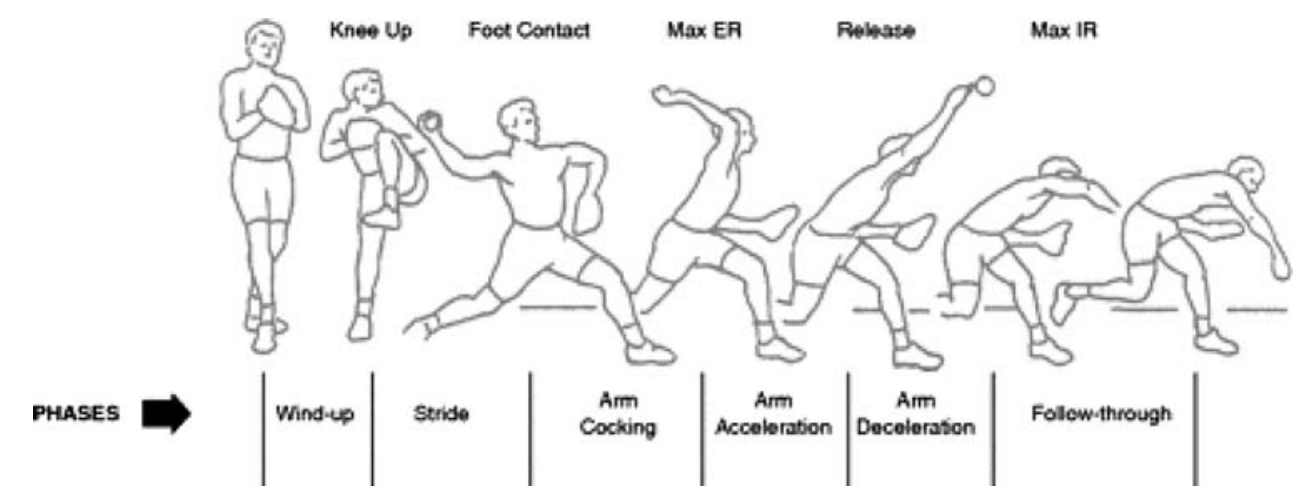




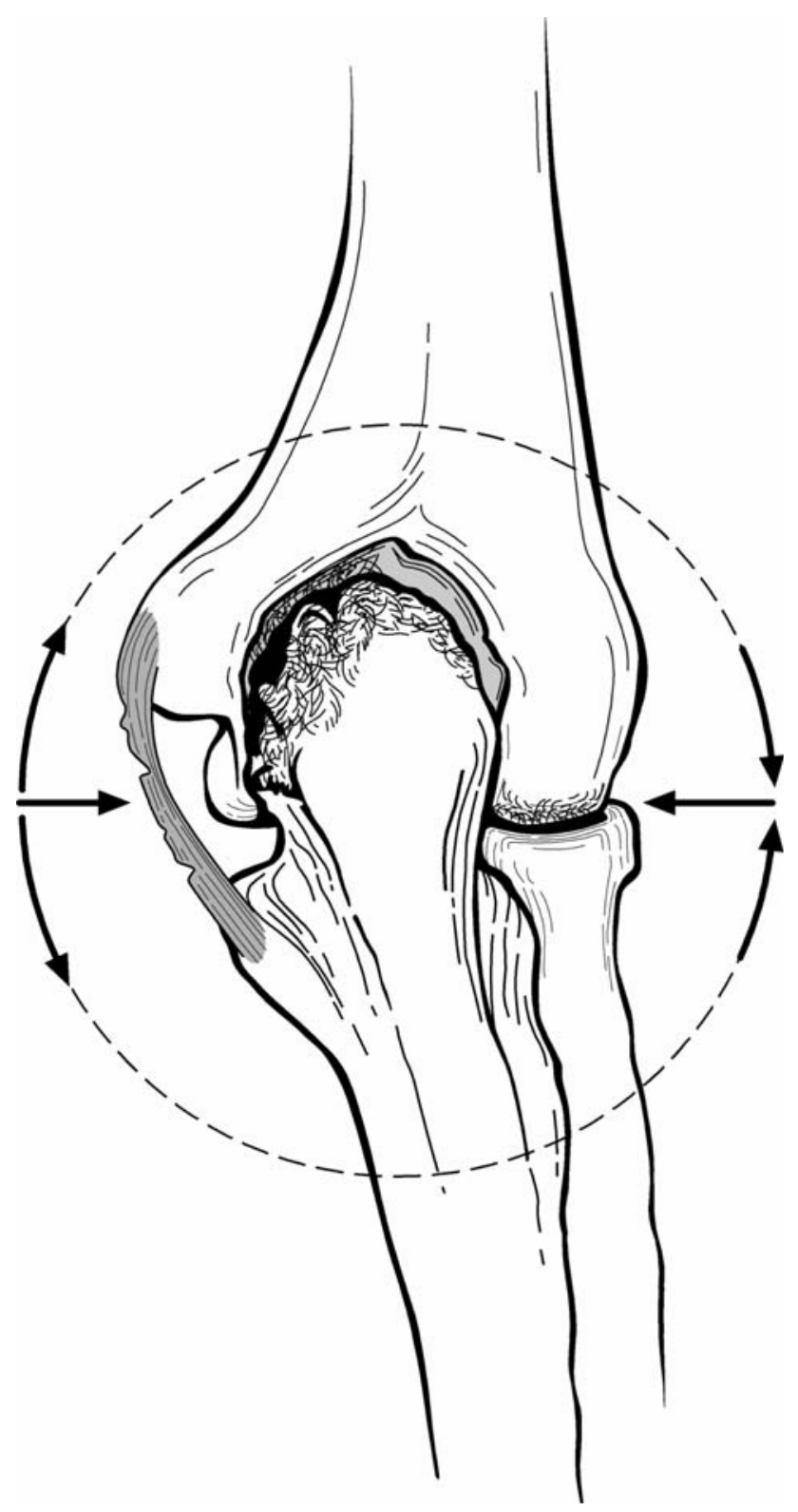

Fig. 2 Illustration of posterior medial osteophytes

olecranon from its fossa as valgus stress is applied. The milking maneuver is performed by either the patient or the examiner pulling on the patient's thumb to create valgus stress with the patients' forearm supinated and elbow flexed beyond $90^{\circ}$. The moving valgus stress test is a modification of the milking maneuver where valgus stress is applied while the elbow is moved through an arc of flexion and extension (Fig. 3). For both tests, the subjective feeling of apprehension, instability, or localized pain to the MCL indicates MCL injury. Physical examination should also assess the degree of extension loss. Pain may be elicited in the posterior compartment with pronation, valgus, and extension forces indicating valgus extension overload. The presence of a palmaris longus should be

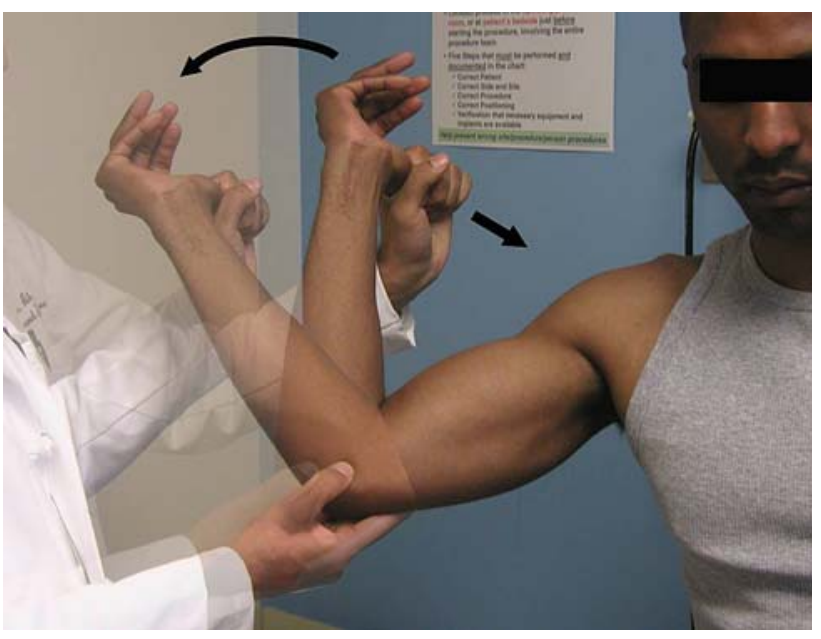

Fig. 3 Moving valgus stress test with arrows indicated examiner applying valgus stress while moving the elbow form flexion to extension (From Ahmad, Orthopaedic Knowledge Update: Shoulder and Elbow 3:Athletic Elbow Injuries in the Throwing Athlete Elbow, AAOS.)

ascertained if MCL reconstruction is anticipated. The ulnar nerve should be palpated for possible subluxation and Tinel's sign over the cubital tunnel should be elicited.

Arthroscopy has been studied to ascertain its utility in diagnosing MCL injury. Of the available studies, a difference in the ability to visualize the AOL was observed; however, all reliably visualized the POL [17-19]. Although the AOL is not directly visualized well arthroscopically, arthroscopic valgus stress test can indicate MCL insufficiency. Arthroscopy is not routinely used to diagnose MCL injuries. Its use is limited to patients with persistent medial elbow pain deemed difficult to diagnose by an orthopedictrained upper extremity specialist.

\section{Imaging studies}

Anterior-posterior, lateral, and axial views of the elbow are assessed for joint space narrowing, osteophytes, and loose bodies. Valgus stress radiographs may be used to measure medial joint line opening. Opening greater than $3 \mathrm{~mm}$ has been considered diagnostic of valgus instability [1,20,21]. However, mild increased valgus elbow laxity has been observed in uninjured, asymptomatic dominant elbows of professional baseball pitchers when compared with their non-dominant elbow [22]. Computed tomography and MRI are useful tools to further define loose bodies and osteophytes. Conventional MRI may be capable of identifying thickening within the ligament from chronic injury or more obvious full thickness tears. MR arthrography enhanced with intra-articular gadolinium improves the diagnosis of partial undersurface tears [23]. Plain MRI sensitivity is 
$57 \%$ and specificity is $100 \%$, while MR arthrography is 92\% sensitive and $100 \%$ specific [24]. Therefore the preferred imaging technique is MR enhanced with intraarticular gadolimium contrast, high field closed magnet, and narrow slice images. A full thickness tear of the MCL from its attachment to the medial humeral epicondyle is shown in Fig. 4.

Dynamic ultrasonography has recently been studied as a means to evaluate the MCL and is capable of detecting increased laxity with valgus stress [25]. Also, Miller et al. [26] used sonography without valgus stress in eight baseball pitchers and concluded that sonography is a useful modality for detecting MCL pathology. Advantages of ultrasound are that it is non-invasive, inexpensive, and dynamic. A disadvantage lies in the dependency on operator experience.

\section{Indications/contraindications}

Treatment decisions require consideration of the individual athlete's demands and the degree of MCL injury. Nonoperative treatment includes a 6-week period of rest from throwing and flexor pronator strengthening [27]. If the patient is asymptomatic and has a normal exam, then return to throw with optimizing throwing mechanics is begun. Rettig et al. [28] demonstrated a $42 \%$ return to the same level of play with an average return at 24.5 weeks with

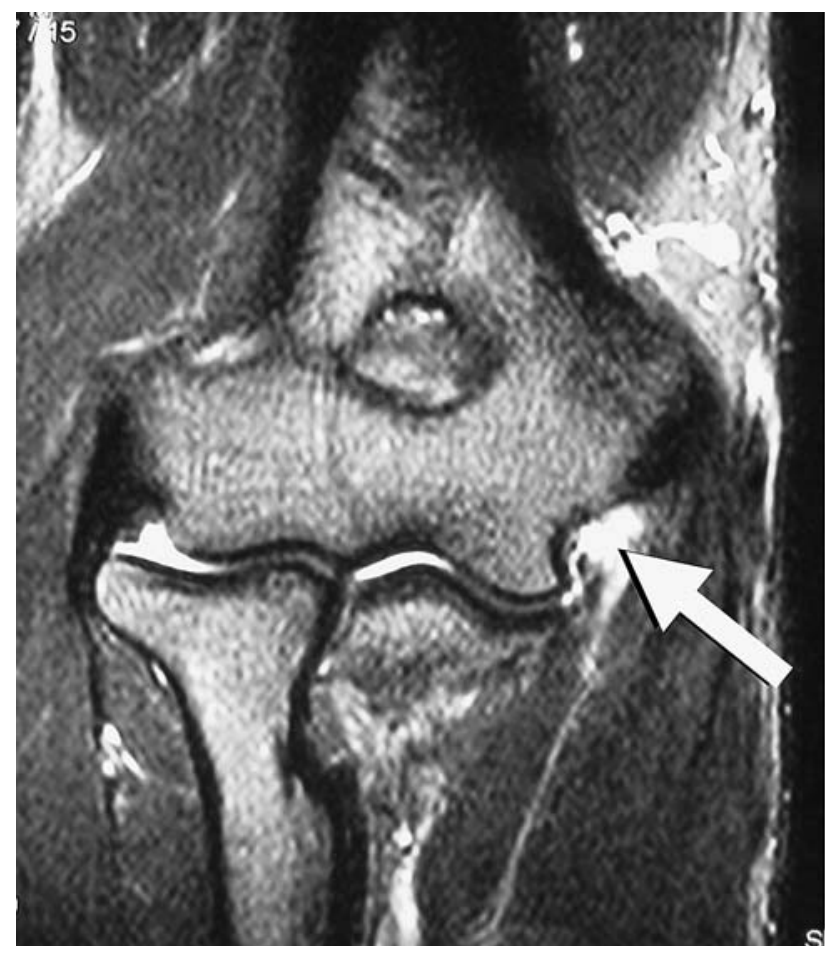

Fig. 4 MRI with arrow indicating MCL tear non-operative management. Primary care providers should consider consultation by an orthopedist when patients have failed an initial compliant course of physical therapy.

Indications for MCL reconstruction require an accurate diagnosis of the MCL injury with proper history, physical exam, and imaging studies. Patients with a diagnosis of MCL insufficiency that fail non-operative treatment are candidates for MCL reconstruction. Patients who wish to continue throwing, have failed non-operative treatment, have an accurate diagnosis, and are willing to participate in the lengthy rehabilitation are candidates for surgical reconstruction.

Contraindications to surgical MCL reconstruction include those athletes with asymptomatic tears that most commonly exist in patients with little valgus demands on the elbow. Some patients who do not wish to continue throwing or who cannot participate in the extensive rehabilitation are contraindicated. Patients with co-existing ulnohumeral or radiocapitellar arthritis considering MCL reconstruction should be informed of the possibility of continued or worsening pain following reconstruction.

\section{Surgical techniques}

The original MCL reconstruction (Fig. 5)

Jobe [1] developed the original MCL reconstruction and described the technique with initial results. The technique

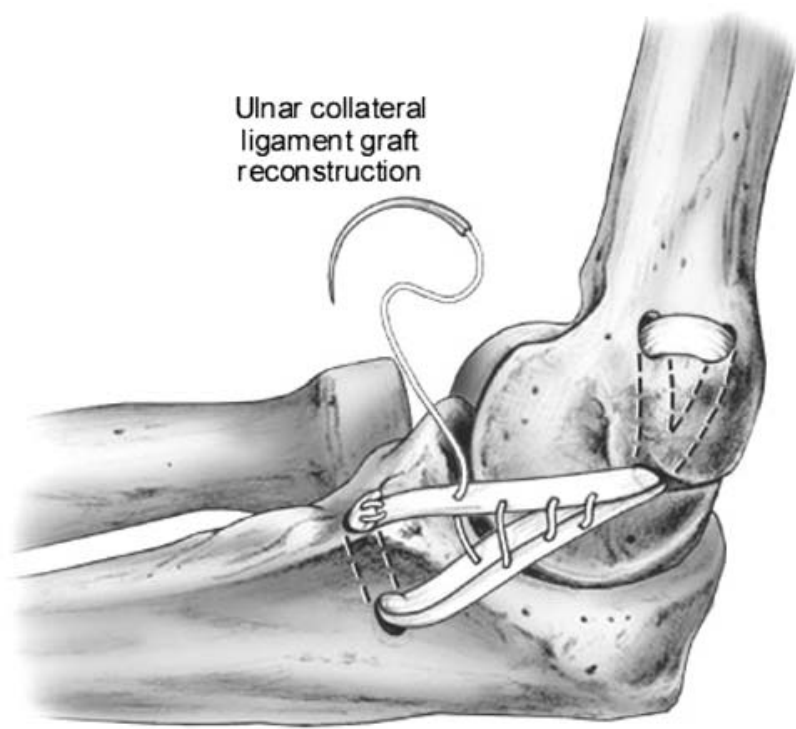

Fig. 5 The original MCL reconstruction technique as described by Jobe demonstrating detachment of the flexor-pronator mass, transposition of the ulnar nerve, and bone tunnels directed posterior on the humeral epicondyle (Reprinted with permission from Safran M, Ahmad CS, ElAttrache: ulnar collateral ligament of the elbow. Arthroscopy 2005;21:1381-1395.) 
used a tendinous detachment and reflection of the flexorpronator mass, submuscular transposition of the ulnar nerve, and creation of humeral tunnels that penetrated the posterior humeral cortex. While his technique was successful in returning elite throwers back to their pre-injury level, it was technically demanding and there was a high complication rate, up to $21 \%$, involving the ulnar nerve problems [1]. Refinements of this procedure will be discussed below. In brief, better outcomes have been obtained with fewer complications by using a muscle splitting approach and eliminating the ulnar nerve transposition [2].

\section{Modified Jobe technique}

A skin incision centered over the medial epicondyle. The sensory branches of the medial antebrachial cutaneous nerve are consistently identified. The flexor-pronator mass is split by incising the raphe from the medial epicondyle to the sublime tubercle (Fig. 6). The flexor muscle mass is separated from the ulnar collateral ligament. The ligament is inspected (Fig. 7) and palpated while a valgus stress is applied. A longitudinal split is made in the ligament and valgus stress with the elbow at $30^{\circ}$ flexion will reveal opening of the ulnohumeral articulation if the MCL is insufficient. Converging drill holes are made in the ulna.

A drill hole is made at the site of the anatomic origin of the anterior bundle of MCL on the medial epicondyle that does not penetrate the posterior cortex. A drill hole is placed just anterior to the epicondylar attachment of the medial intermuscular septum and directed to communicate with the drill hole in the epicondyle. A second drill hole is made in the anterosuperior surface of the epicondyle approximately $1 \mathrm{~cm}$ from the previous hole.

The palmaris longus from the ipsilateral arm is harvested through a series of small transverse incisions. The graft is passed through the proximal ulnar bone tunnel and medial epicondyle as configured in Fig. 8. The graft is

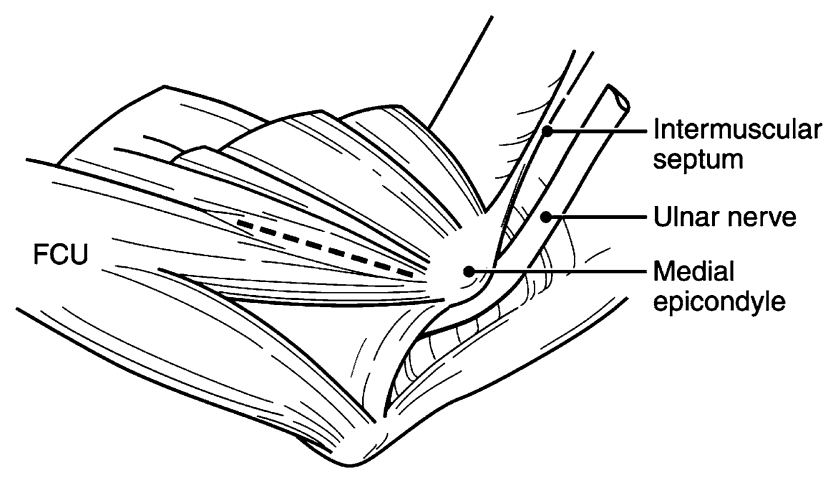

Fig. 6 Location of muscle split in flexor-pronator mass (Reprinted with permission from Conway JE. The DANE TJ procedure for elbow medial ulnar collateral ligament insufficiency. Techniques in Shoulder and Elbow Surgery 2006;7(1):36-43.)

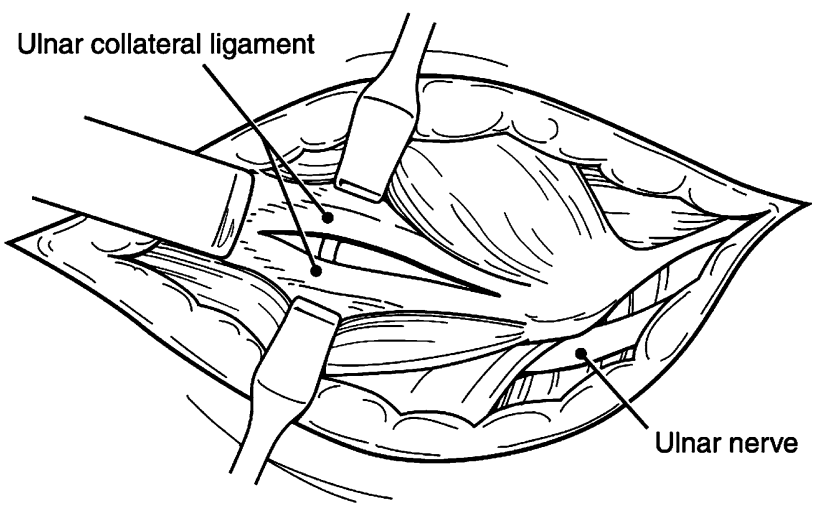

Fig. 7 Muscle split approach and ulnohumeral gapping demonstrated after MCL incised

tensioned with the elbow flexed with an applied varus stress. The ulnar side of the graft is sutured to the remnants of the ulnar collateral ligament adjacent to the sublime tubercle. The proximal limb of the graft is sutured to the medial intermuscular septum outside the drill hole on the superior surface of the epicondyle. The native ligament is then repaired over the graft with simple sutures placed. The muscle fascia is repaired and the skin is closed.

\section{Docking technique}

The docking technique is a modification of the Jobe technique that simplifies graft passage, tensioning, and fixation by using sutures to control to limbs passed through the humerus (Fig. 8). The docking technique modification utilizes the muscle splitting approach with tunnel creation on

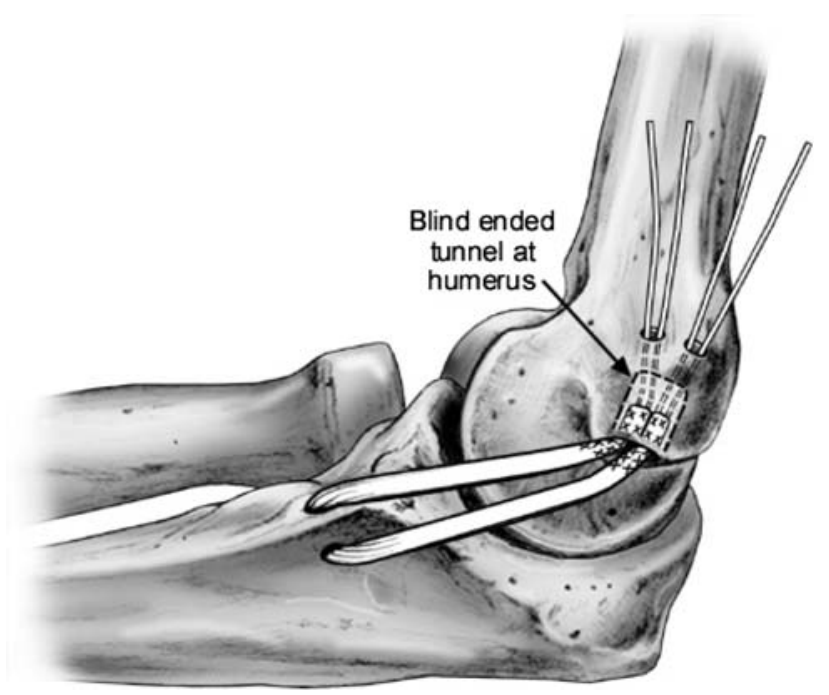

Fig. 8 The docking technique creates exit holes superior to the inferior humeral tunnel for tensioning graft-controlling sutures (Reprinted with permission from Safran M, Ahmad CS, ElAttrache: ulnar collateral ligament of the elbow. Arthroscopy 2005;21:13811395.) 
the ulna similar to the Jobe technique. The humeral tunnel position is located in the anterior half of the medial epicondyle at the anatomic insertion of the native MCL similar to the Jobe technique. The graft is fashioned to an exact length to fit inside the humeral tunnel. The free ends of the graft are controlled with sutures that are passed through the two exiting tunnels and tied over a bony bridge.

\section{Hybrid interference screw fixation technique (DANE TJ)} (Fig. 9)

The new technique of MCL reconstruction achieves ulnar sided fixation in a single-bone tunnel with an interference screw and humeral fixation using the docking technique [29]. This technique is less technically demanding since the required number of drill holes is reduced. This reduces the chance for surgical error. Less dissection through a muscle splitting approach is afforded since only a single central tunnel is required rather than two tunnels with an intervening bony bridge on the ulna. Less dissection reduces the amount of inflammation secondary to surgical trauma. With a single tunnel, the posterior ulnar tunnel which is in closest proximity to the ulnar nerve is avoided. Finally, graft passage is less difficult with an interference screw, which is a screw placed between the bone and the graft to afford strong fixation, in a single tunnel.

\section{Rehabilitation}

The elbow is immobilized in a splint for 10 days to allow the skin and soft tissues to heal. Then active wrist, elbow,

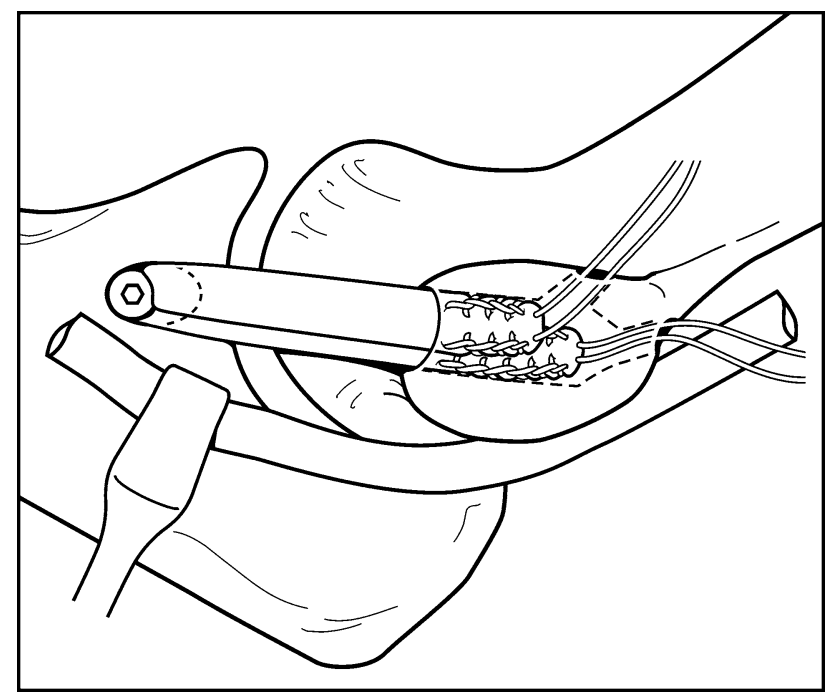

Fig. 9 Hybrid technique of UCL reconstruction with interference screw fixation on ulnar side and docking fixation on humeral side (Reprinted with permission from Conway JE. The DANE TJ procedure for elbow medial ulnar collateral ligament insufficiency. Techniques in Shoulder and Elbow Surgery 2006;7(1):36-43.) and shoulder range of motion exercises are initiated. After 4-6 weeks, strengthening exercises are begun while avoiding valgus stress until 4 months postoperative. At 4 months postoperative, the patient begins a throwing program initially with ball toss of $30-40 \mathrm{ft}$, two to three times a week for about $15 \mathrm{~min}$. At 5 months the patient may increase the tossing distance to $60 \mathrm{ft}$, and at 6 months the patient may perform throwing lightly from the wind-up. At 7 months a graduated program of range of motion, strengthening, and total-body conditioning exercises is performed. Throwers and pitchers are limited to throw onehalf speed, while gradually increasing the duration of their session to 25-30 min. Pitchers are permitted to throw from the pitching mound and progress to $70 \%$ of maximum velocity during the eighth or ninth month. Over the next 2-3 months, the duration of throwing sessions and velocity are slowly increased to simulate a game situation. Throwing in competition is permitted at 1 year if the shoulder, elbow, and forearm are pain-free while throwing and full strength and range of motion have returned. Throughout the rehabilitation phase, careful supervision and focus on body and throwing mechanics should be emphasized. Eighteen months may be required to regain preoperative ability and competitive level with accurate ball control. Relatively shorter periods are required for other player positions or overhead sports.

\section{Outcome}

Many of the intricate details of MCL reconstruction should be discussed by an orthopedic-trained upper extremity specialist. However, for completeness the primary care providers can advise patients that patient satisfaction is very high after MCL reconstruction by a specialist in the appropriate patient. MCL reconstruction is technically demanding with regard to limit muscular dissection and avoiding ulnar nerve injury. Furthermore, achieving graft isometry, adequate graft tension, and secure graft fixation remain challenging while optimizing graft-healing biology. Reports of clinical outcome for MCL reconstruction have been variable with $68-93 \%$ having good to excellent results. In a recent study by Thompson et al. [21], 93\% of patients who had no previous elbow surgery had excellent results at 2-year follow-up. The improved outcomes of recent studies can be attributed to minimize dissection of the flexor-pronator mass and handling of the ulnar nerve.

Many studies have been done which demonstrate the ability of elite athletes to return to the pre-injury level of play after MCL reconstruction [29-31]. Furthermore, the incidence of ulnar neuritis seen in earlier procedures has decreased dramatically when an ulnar nerve transposition is performed. The newer surgical modifications such as the docking technique and use of the interference screw are 
constantly being studied. To date, these techniques have yielded excellent results [32-35].

\section{Summary}

MCL injury is a common cause of medial elbow pain. A thorough summation of available history, physical exam, and imaging is required to reach an accurate diagnosis. Recent modifications of the original surgical procedure yield improved clinical results. Prevention strategies are needed to advance our knowledge in this area. Patients should have a course of non-operative therapy as a large percentage of patients will respond. Those who fail conservative management should be referred to an orthopedist for further evaluation.

Open Access This article is distributed under the terms of the Creative Commons Attribution Noncommercial License which permits any noncommercial use, distribution, and reproduction in any medium, provided the original author(s) and source are credited.

\section{References}

1. Jobe FW, Stark H, Lombardo SJ. Reconstruction of the ulnar collateral ligament in athletes. J Bone Joint Surg Am. 1986;68(8): 1158-63.

2. Chen FS, Rokito AS, Jobe FW. Medial elbow problems in the overhead-throwing athlete. J Am Acad Orthop Surg. 2001;9(2): 99-113.

3. Schickendantz MS. Diagnosis and treatment of elbow disorders in the overhead athlete. Hand Clin. 2002;18(1):65-75.

4. Cain EL Jr, Dugas JR, Wolf RS, Andrews JR. Elbow injuries in throwing athletes: a current concepts review. Am J Sports Med. 2003;31(4):621-35.

5. Sellards R, Kuebrich C. The elbow: diagnosis and treatment of common injuries. Prim Care. 2005;32(1):1-16.

6. Safran M, Ahmad C, Elattrache NS. Ulnar collateral ligament of the elbow. Arthroscopy. 2005;21(11):1381-95.

7. Nassab PF, Schickendantz MS. Evaluation and treatment of medial ulnar collateral ligament injuries in the throwing athlete. Sports Med Arthrosc. 2006;14(4):221-31.

8. Petty DH, Andrews JR, Fleisig GS, Cain EL. Ulnar collateral ligament reconstruction in high school baseball players: clinical results and injury risk factors. Am J Sports Med, 2004;32(5): 1158-64.

9. O'Driscoll SW, Jaloszynski R, Morrey BF, An KN. Origin of the medial ulnar collateral ligament. J Hand Surg Am. 1992;17(1): 164-8.

10. Callaway GH, Field LD, Deng XH, Torzilli PA, O'Brien SJ, Altchek DW, et al. Biomechanical evaluation of the medial collateral ligament of the elbow. J Bone Joint Surg Am. 1997;79(8): 1223-31.

11. Hotchkiss RN, Weiland AJ. Valgus stability of the elbow. J Orthop Res. 1987;5(3):372-7.

12. Regan WD, Korinek SL, Morrey BF, An KN. Biomechanical study of ligaments around the elbow joint. Clin Orthop Relat Res. $1991 ;(271): 170-9$.

13. Morrey BF, An KN. Articular and ligamentous contributions to the stability of the elbow joint. Am J Sports Med. 1983;11(5): 315-9.
14. Werner SL, Fleisig GS, Dillman CJ, Andrews JR. Biomechanics of the elbow during baseball pitching. J Orthop Sports Phys Ther. 1993;17(6):274-8.

15. Kooima CL, Anderson K, Craig JV, Teeter DM, van Holsbeeck M. Evidence of subclinical medial collateral ligament injury and posteromedial impingement in professional baseball players. Am J Sports Med. 2004;32(7):1602-6.

16. Andrews JR, Timmerman LA. Outcome of elbow surgery in professional baseball players. Am J Sports Med. 1995;23(4):40713.

17. Field LD, Altchek DW. Evaluation of the arthroscopic valgus instability test of the elbow. Am J Sports Med. 1996;24(2):17781.

18. Field LD, Callaway GH, O'Brien SJ, Altchek DW. Arthroscopic assessment of the medial collateral ligament complex of the elbow. Am J Sports Med. 1995;23(4):396-400.

19. Timmerman LA, Andrews JR. Histology and arthroscopic anatomy of the ulnar collateral ligament of the elbow. Am J Sports Med. 1994;22(5):667-73.

20. Conway JE, Jobe FW, Glousman RE, Pink M. Medial instability of the elbow in throwing athletes. Treatment by repair or reconstruction of the ulnar collateral ligament. J Bone Joint Surg Am. 1992;74(1):67-83.

21. Thompson WH, Jobe FW, Yocum LA, Pink MM. Ulnar collateral ligament reconstruction in athletes: muscle-splitting approach without transposition of the ulnar nerve. J Shoulder Elbow Surg. 2001;10(2):152-7.

22. Ellenbecker TS, Mattalino AJ, Elam EA, Caplinger RA. Medial elbow joint laxity in professional baseball pitchers. A bilateral comparison using stress radiography. Am J Sports Med. 1998; 26(3):420-4.

23. Hill NB Jr, Bucchieri JS, Shon F, Miller TT, Rosenwasser MP. Magnetic resonance imaging of injury to the medial collateral ligament of the elbow: a cadaver model. J Shoulder Elbow Surg. 2000;9(5):418-22.

24. Munshi M, Pretterklieber ML, Chung CB, Haghighi P, Cho JH, Trudell DJ, et al. Anterior bundle of ulnar collateral ligament: evaluation of anatomic relationships by using MR imaging, MR arthrography, and gross anatomic and histologic analysis. Radiology. 2004;231(3):797-803.

25. Sasaki J, Takahara M, Ogino T, Kashiwa H, Ishigaki D, Kanauchi Y. Ultrasonographic assessment of the ulnar collateral ligament and medial elbow laxity in college baseball players. J Bone Joint Surg Am. 2002;84A(4):525-31.

26. Miller TT, Adler RS, Friedman L. Sonography of injury of the ulnar collateral ligament of the elbow-initial experience. Skeletal Radiol. 2004;33(7):386-91.

27. Ahmad CS, Park KN, Elattrache KN. Elbow medial ulnar collateral ligament insufficiency alters posteromedial olecranon contact. Am J Sports Med. 2004;32(7):1607-12.

28. Rettig AC, Sherrill C, Snead DS, Mendler JC, Mieling P. Nonoperative treatment of ulnar collateral ligament injuries in throwing athletes. Am J Sports Med. 2001;29(1):15-7.

29. Koh JL, Schafer MF, Keuter G, Hsu JE. Ulnar collateral ligament reconstruction in elite throwing athletes. Arthroscopy. 2006; 22(11):1187-91.

30. Azar FM, Andrews JR, Wilk KE, Groh D. Operative treatment of ulnar collateral ligament injuries of the elbow in athletes. Am J Sports Med. 2000;28(1):16-23.

31. Rohrbough JT, Altchek DW, Hyman J, Williams RJ 3rd, Botts JD. Medial collateral ligament reconstruction of the elbow using the docking technique. Am J Sports Med. 2002;30(4): $541-8$.

32. Dodson CC, Thomas A, Dines JS, Nho SJ, Williams RJ 3rd, et al. Medial ulnar collateral ligament reconstruction of the elbow in throwing athletes. Am J Sports Med. 2006;34(12):1926-32. 
33. Paletta GA Jr, Wright RW. The modified docking procedure for elbow ulnar collateral ligament reconstruction: 2-year follow-up in elite throwers. Am J Sports Med. 2006;34(10):1594-8.

34. Ahmad CS, Lee TQ, ElAttrache NS. Biomechanical evaluation of a new ulnar collateral ligament reconstruction technique with interference screw fixation. Am J Sports Med. 2003;31(3):332-7.
35. Dines JS, ElAttrache NS, Conway JE, Smith W, Ahmad CS. Clinical outcomes of the DANE TJ technique to treat ulnar collateral ligament insufficiency of the elbow. Am J Sports Med. 2007;35(12):2039-44. 